\title{
COMPARATIVE RESEARCH OF THE SUB- GINGIVAL IMPRESSION QUALITY BY FIXED PROSTHESIS USING ONE AND DOUBLE CORD RETRACTION TECHNIQUE
}

\author{
Metodi Abadzhiev \\ Prosthodontic Department, Faculty of Dental Medicine, \\ Medical University - Varna
}

\section{SUMMARY:}

The accurate impression of every detail of the prosthetic area is of extreme importance for the successful prosthetic restorations. One of the problems appearing in the process of fixed prosthetic restoration is the accurate impression of the marginal details. Contemporary dentistry uses hydrophilic impression materials (polyeter, A-silikone) allowing operation in damp environment. However, appropriate gingival retraction of sulcus gingivalis is of utmost importance, as even the most modern nano impression materials are unable to guarantee an accurate marginal detail. A high-quality impression that provides the necessary marginal detail is not only required for good fit, but also for optimal esthetic results.

Key words: Polyeter impression material, A-silikon, retraction cords

\section{PURPOSE:}

The present paper focuses on the necessity of a second retraction cord used to ensure dryness of the gingival sulcus, thus allowing unimpeded ingress of the impression material and exact impression of the marginal detail.

\section{INTRODUCTION:}

The retraction of the gingival sulcus is a long established technique. Today we can sey that is a management of soft tissue before an impression procedure.In the past retraction was implemented using various techniques among which event the application of cuprum ferrule. Later on retraction cords gained wide application. Notwithstanding the wide variety of retraction materials, the use of retraction cords is still considered 'the classic' technique for gingival retraction. (fig. 1.) Saturated with $10 \%$ aluminum chloride or $15.5 \%$ ferric sulfate, the retraction cords ensure dryness in the ginvival pocket and let the impression material cover the desired area. The procedure of the two-phase two-layer impression technique requires application of the retraction cord, impression taking with the first putty impression layer, removal of the retraction cord, and taking the second impression with fine impression material. Unfortunately the removal of the cord might provoke a drop of blood or some gingival fluid. With a greater number of prepared teeth the process of air drying is harder. In other cases, with a deeper subgingival preparation, after removing the cord, the sulcus 'closes' not allowing the ingress of the impression material in the subgingival area. (fig. 2.) Such cases necessitate the use of a second retraction cord. The technique involves the application of a second cord, thicker in size. Before making the final impression the cord applied last is removed. One cord is preserved in the sulcus to keep the area dry and to prevent the retracted gingival margin to 'return' onto the prepared shoulder. (fig. 3.)

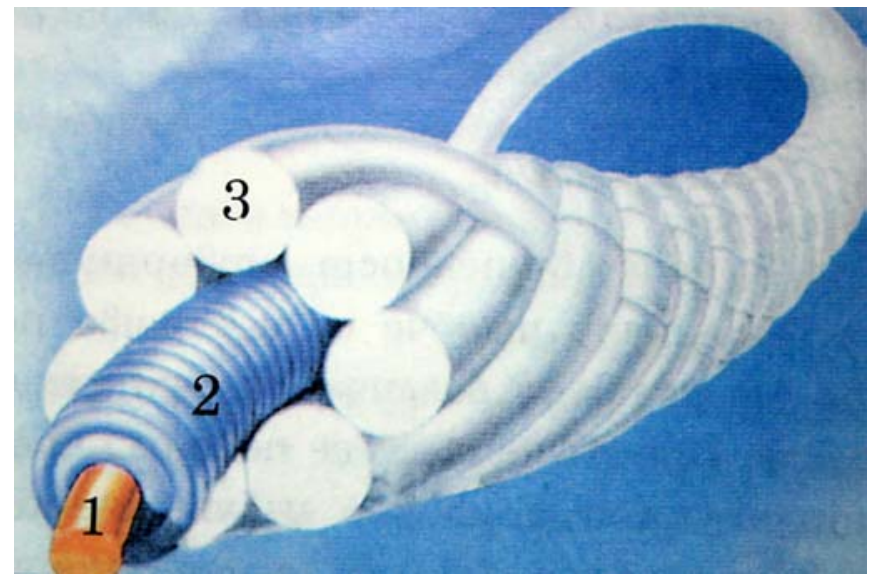

Fig. 1. One of the design ot retractions cords. 

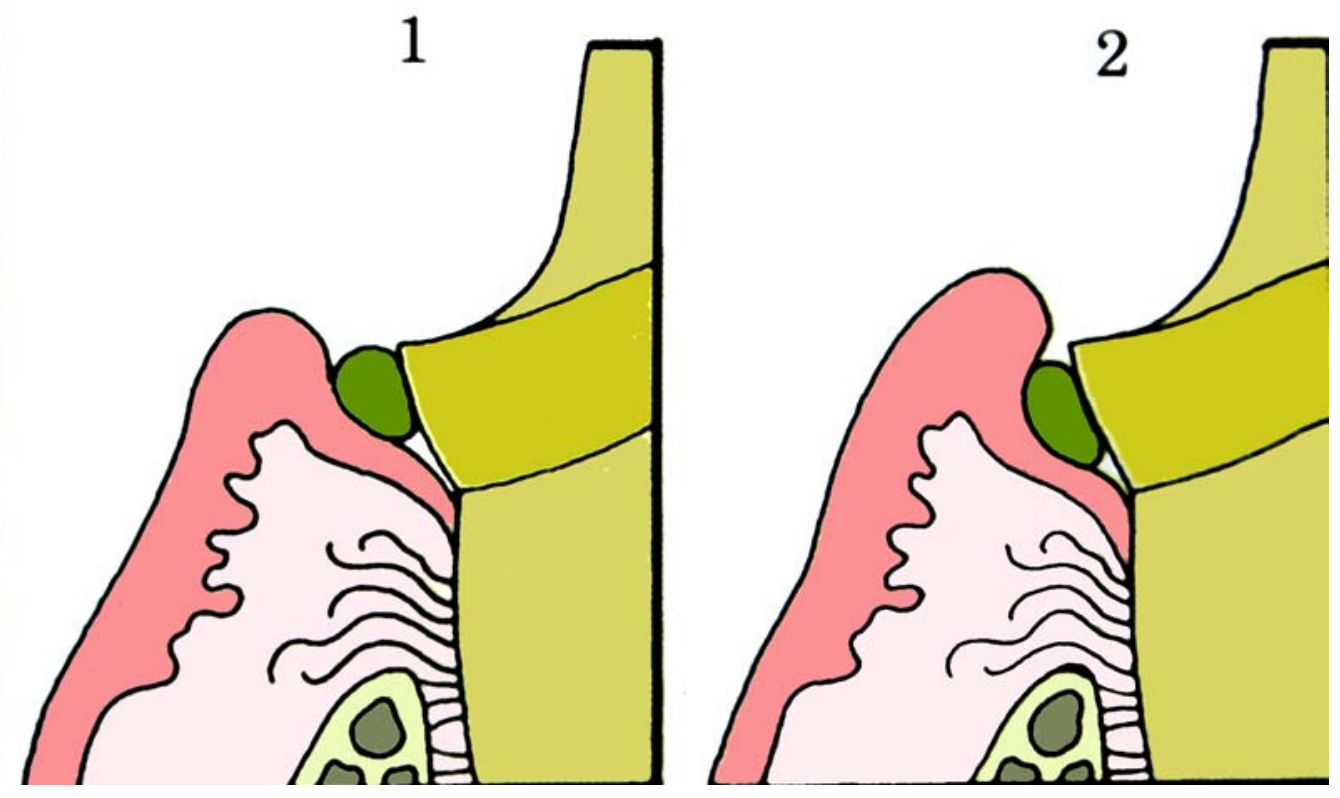

Fig. 2. One cord retractions technique.

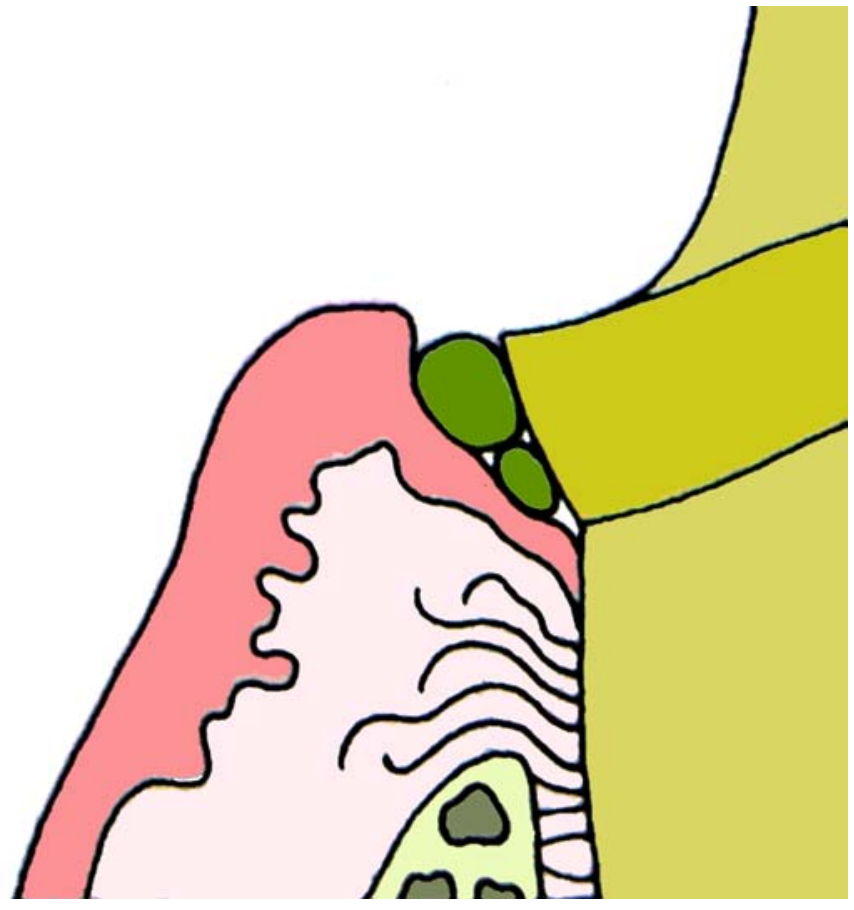

Fig. 3. Double cord retractions technique.

\section{METHODS AND MATERIALS:}

ULTRAPAK Retraction cords of four different sizes, and ULTRAPAK Retraction gel. Polyeter impression material IMPREGUM for machine mixing with Pentamix and Impegym Duo Soft produced by 3M ESPE, (fig. 4.) as well as adhesive varnish product of the same company. Single-phase and double-layer (double-mix) impression technique. Standard metal impression trays 60 impressions of total 103 prosthetic restorations with 325 supported teeth with subgingival shoulder preparation. 30 of the impressions (103 supports) were implemented with one cord and 30 (222 supports) were implemented with two retraction cords. Anonimous enquiry provided an objective picture of the impression quality evaluated by 10 different dental technishians. The possible answers were graded as follows: excellent, good, poor. The assessment was implemented using a Mantis Vision laboratory microscope and documented with a digital camera CANON EOS 300 D fitted with a 100 macro lens and ring flash MACRO RING LITE MR - 14 EX.

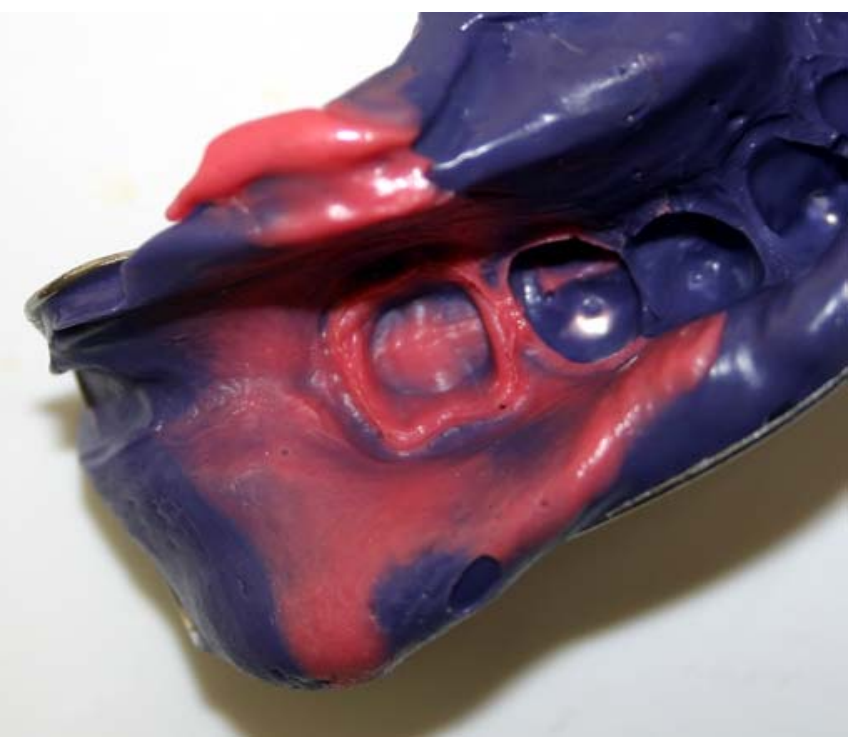

Fig. 4. IMPREGUM impressine. 


\section{RESULTS:}

43 of the supports incorporated in the first test group were assessed as poor (41,7\%) 36 were evaluated as good $(36 \%)$ and 24 obtained an excellent grade $(23 \%)$. In the second test group 8 were assessed as poor (3,6\%), 61- as good $(27,4 \%)$, and 183 were evaluated as being excellent $(82,4 \%)$. The obtained results clearly obviate the advantages of the second retraction technique.

\section{DISCUSSION:}

The offered technique, like many other dental techniques, should be patient-based and applied whenever the individual treatment necessitates, or allows it. The main criteria on the impression technique to be applied incorporate two parameters: the parodont condition of the prepared teeth and the depth of the gingival sulcus. Occasionally a gingival pocket may not be deep enough to allow the application of a second retraction cord. Other cases necessitate the use of several cords (especially approximally in the papilus area). In all cases special care is to be taken and the thin periodontal tissue should be treated very gently. (fig.5.)

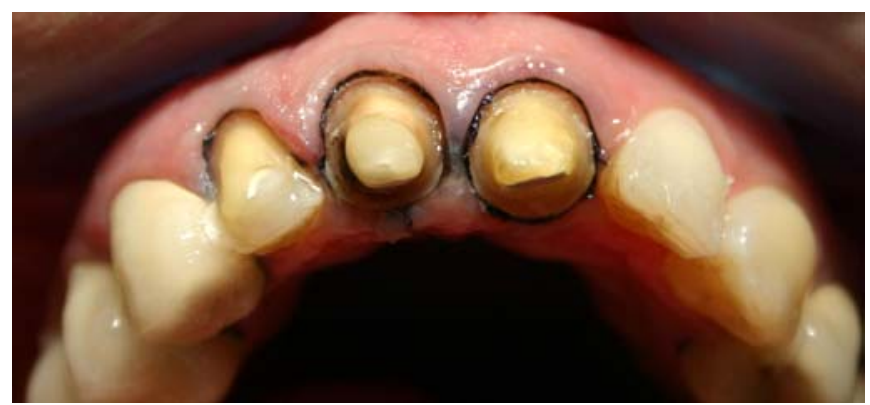

Fig. 5. A clinical situation after putting the second cord.

\section{CONCLUSIONS:}

The accuracy of the impression taken in the prosthetic area is extremely important both for the health and the esthetics of the treated patients. When a second retraction cord is applied it retracts the gingival sulcus permanently, keeps it dry and allows a deep ingress of the impression material. Such a technique might take some more time and incur additional expenses, but the accuracy of the impression and the esthetic and prophylactic prosthetics make it worth any additional expenditure. Double cord retractions technique should be a standart in preparation of soft and hard tissue for impressions in fixed prosthodontie.

\section{REFERENCES:}

1. Impression materials and impression methods in fixed prosthodontics, Christo Kisov, INDEX - Sofia, 1998

2.Using the double-cord packing technique of tissue retraction for making crown impressions.Cloyd S, Puri S.Dent Today. 1999 Jan; 18(1):54-9.

3. Clinical trial of gingival retraction cords. Jokstad A. J Prosthet Dent. 1999 Mar;81(3):258-61

4.Tooth preparations for all ceramic and metal-ceramic crowns, Christo Kisov,
INDEX - Sofia, 2000.

5. Effects of diameter, chemical impregnation and hydration on the tensile strength of gingival retraction cords.Del Rосно Nieto-Marthnez M., Маupomй G, Barcely-Santana F. J Oral Rehabil. 2001 Dec; 28(12):1094-100.

6. Effects of gingival retraction materials on gingival blood flow. Polat NT, Ozdemir AK, Turgut M.Int J Prosthodont. 2007 Jan-Feb; 20(1):57-62.

7. Effect of different retraction and impression techniques on the marginal fit of crowns. Wцstmann B., Rehmann P., Trost D., Balkenhol M. J Dent. 2008 Jul; 36(7): 508-12. Epub 2008 May 13.

8. A clinical study on the effects of cordless and conventional retraction techniques on the gingival and periodontal health. Al Hamad K. Q., Azar W. Z., Alwaeli H. A., Said K. N. J Clin Periodontol. 2008 Dec;35(12):1053-8.

\author{
Address for correspondence: \\ Metodi Abadzhiev \\ Prosthodontic Department, Faculty of Dental Medicine, Medical University - Varna \\ 63, G. S. Rakovski Str., 9000 Varna, Bulgaria \\ E-mail: mabadjieff@yahoo.com
}

